\title{
Adiponectin and the development of diabetes in patients with coronary artery disease and impaired fasting glucose
}

\author{
Hilla Knobler, Michal Benderly ${ }^{1}$, Valentina Boyko ${ }^{1}$, Shlomo Behar ${ }^{1}$, Zipora Matas ${ }^{2}$, Ardon Rubinstein ${ }^{3}$, \\ Itamar $\operatorname{Raz}^{4}$ and Julio Wainstein ${ }^{5}$ \\ Metabolic Unit, Kaplan Medical Center, Rehovot, Israel, ${ }^{1}$ BIP Study Group Coordinating Center, Neufeld Cardiac Research Institute, Sheba Medical Center, \\ Tel Hashomer, Tel-Aviv, Israel, ${ }^{2}$ Biochemistry Laboratory, Wolfson Medical Center, Holon, Israel, ${ }^{3}$ Metabolic Institute, Tel-Aviv Sourasky Medical Center, \\ Tel-Aviv, Israel, ${ }^{4}$ Israel Diabetes Center, Hadassah Medical Center, Jerusalem, Israel and ${ }^{5}$ Diabetes Center, Wolfson Medical Center, Holon, Israel \\ (Correspondence should be addressed to H Knobler; Email: knobler@inter.net.il)
}

\begin{abstract}
Objectives: Adiponectin has insulin-sensitizing properties, and high adiponectin levels have been shown to be associated with reduced risk of developing diabetes. Patients with coronary artery disease (CAD) have relatively low adiponectin levels and high prevalence of glucose intolerance. The role of adiponectin in predicting the development of diabetes in this high-risk group has not been determined. The study aimed to determine whether baseline adiponectin levels predict the development of diabetes in a group of patients with CAD and impaired fasting glucose (IFG).

Methods: A total of 588 patients who participated in the Bezafibrate Infarction Prevention (BIP) study and who had at baseline fasting glucose of $100-125 \mathrm{mg} / \mathrm{dl}$ were included and followed for $6.2 \pm 1.3$ years. Adiponectin was determined in frozen plasma samples taken at baseline.

Results: Of the patients with IFG at baseline, 256 (44\%) developed diabetes during follow-up. The patients who developed diabetes had at baseline higher body-mass index, fasting glucose, C-reactive protein, triglycerides, homeostatic assessment of insulin resistance (HOMA-IR) and diastolic blood pressure than patients who did not develop diabetes. Adiponectin levels at baseline were significantly lower in patients who developed diabetes than in patients who did not develop diabetes $(P=0.009$, nonparametric Kruskall-Wallis test). An increase of 1 unit of natural logarithm of adiponectin level was associated with a hazard ratio of 0.77 (95\% CI, 0.61-0.96) for diabetes development.

Conclusion: Patients with CAD and IFG have a very high rate of conversion to type 2 diabetes. Even in this high-risk group, high adiponectin levels are associated with reduced risk of developing diabetes.
\end{abstract}

European Journal of Endocrinology 154 87-92

\section{Introduction}

Patients with known coronary artery disease (CAD) have a high prevalence of undiagnosed diabetes mellitus (DM) and glucose intolerance (1-3). In previous study based on data from the Bezafibrate Infarction Prevention (BIP) study, a secondary prevention trial, $20 \%$ of the patients had fasting glucose levels of $\geq 110 \mathrm{mg} / \mathrm{dl}$ (4). These patients were characterized by a higher body-mass index (BMI) and fasting glucose levels, by a lipid profile typical of the metabolic syndrome and by a worse clinical outcome. Impaired fasting glucose (IFG) is a 'prediabetes' state with a high rate of conversion to type $2 \mathrm{DM}$ (5). Recent epidemiologic data have shown that low levels of adiponectin, a cytokine exclusively synthesized in adipose tissue, were associated with higher risk of subsequently developing type $2 \mathrm{DM}(6-8)$. The adiponectin gene is located on chromosome 3q27, where a susceptibility locus for diabetes and the metabolic syndrome has been mapped (9). Although the physiologic role of adiponectin is yet to be fully elucidated, numerous animal studies in recent years have shown that adiponectin increases insulin sensitivity (10-13). Adiponectin was shown to decrease hepatic glucose output and to enhance muscle fat oxidation and glucose transport. In man, adiponectin levels were shown to correlate negatively with BMI, C-reactive protein (CRP), and fasting level of glucose, insulin and triglycerides, and to correlate positively with high-density lipoprotein cholesterol (HDL-C) $(10,14,15)$ Adiponectin levels are also significantly lower in patients with CAD than in control subjects (16), and adiponectin levels are inversely correlated with progression of coronary artery calcification in diabetic and nondiabetic patients (17). The antiatherogenic effects of adiponectin have been attributed partly to its anti-inflammatory properties (12). The low levels of adiponectin observed in 
CAD patients may be linked to the high prevalence of glucose intolerance and increased risk of developing type 2 diabetes. Moreover, adiponectin insulin-sensitizing actions may be altered in CAD patients, many with proinflammatory state, a known risk factor also for type 2 DM development. The role of adiponectin in regulating glucose homeostasis in this high-risk group has not been determined. The aim of the current study was to assess in a group of CAD patients who participated in the BIP study, with IFG at baseline, whether adiponectin levels predict the development of subsequent type 2 DM.

\section{Subjects and methods}

\section{Subjects and design}

The BIP study, a secondary prevention, prospective, double-blind study comparing bezafibrate (400 mg daily) to placebo in patients with established CAD, was conducted in 18 cardiology departments in Israel. The BIP study design and results were previously described (18). Inclusion criteria included age of 4574 years, history of myocardial infarction of 6 months to 5 years before enrollment, and/or stable angina pectoris confirmed by coronary angiography and/or radionuclear studies or standard exercise tests. A total of 3122 patients were included and followed for $6.2 \pm 1.3$ years, with routine visits to the clinics, including blood testing for fasting glucose and lipid levels, every 4 months. Patients were diagnosed as having IFG when they had on two occasions during the pre-randomization period, fasting baseline glucose levels of $100-125 \mathrm{mg} / \mathrm{dl}$ (19), had no prior diagnosis of DM or were not receiving DM treatment. During follow-up, patients who had fasting blood glucose of $>126 \mathrm{mg} / \mathrm{dl}$ on two occasions, or who started to receive DM treatment were diagnosed as having DM. A total of 617 patients were diagnosed with IFG by these criteria. In 29 patients (12 in the group who later developed DM and 17 in the group who did not develop DM), no plasma samples were available for adiponectin measurements. Thus, 588 patients $(95 \%)$ were included in the final analysis.

\section{Laboratory methods}

Detailed data on laboratory methods were given in a previous report (18). Briefly, blood samples collected in the 18 participating centers were transported in cooled containers to a central laboratory. Blood samples were drawn after at least $12 \mathrm{~h}$ of fasting. Laboratory measurements of lipid, glucose and fibrinogen levels were made by standard automated procedures with commercially available kits (Roche). Fasting glucose levels were determined in baseline samples by the GOD-PAPP method with a BM/Hitachi 717/911 analyzer. For the purpose of this study, plasma samples that had been taken at baseline and stored as frozen aliquots at $-70{ }^{\circ} \mathrm{C}$ were thawed and assayed for insulin and high-sensitive CRP with the Immulite 2000 analyzer (Diagnostics Products Corporation, Los Angeles, CA, USA). We used the manufacturer's solid-phase chemiluminescent immunometric assay. Adiponectin in frozen baseline plasma aliquots was determined by commercial sandwich enzyme immunoassay (R\&D Systems, Minneapolis, MN, USA). The following formula was used: homeostatic assessment of insulin resistance $($ HOMA-IR $)=$ fasting insulin $(\mathrm{U} / \mathrm{l}) \times$ fasting glucose $(\mathrm{mmol} / \mathrm{l}) / 22.5$.

\section{Statistical analysis}

Data were analyzed with SAS software, Version 8.2 (SAS Institute, Cary, NC, USA). Comparisons of dichotomous variables and normally distributed continuous variables were done by the chi-square test and Student's t-test respectively. Geometric means were used for triglycerides, insulin, CRP and adiponectin to correct for their skewed distribution. Non-normally distributed variables were compared by the nonparametric Kruskal-Wallis test, and they were log transformed for further analysis. Spearman's rank correlation coefficients were computed for the association between adiponectin levels and other clinical variables. In the correlation analysis, patients were stratified according to BMI of $<30 \mathrm{~kg} / \mathrm{m}^{2}$ or $\geq 30 \mathrm{~kg} / \mathrm{m}^{2}$, on the basis of previous data showing that the relationship of adiponectin with other clinical variables is influenced by the coexistence of obesity (20). Age and multivariable adjusted hazard of developing diabetes were computed with the Cox proportional hazard model to account for differences in length of follow-up and correlation of covariates. The variables included in the analysis were chosen from previous data (10); they included age, adiponectin (log-transformed), BMI, glucose, HDL-C, triglycerides (log-transformed), HOMA-IR, CRP (log-transformed), and systolic and diastolic blood pressure. The variables were introduced into the models as continuous variables. Model performance was assessed with C-statistics, the area under the receiving operating curve.

\section{Results}

Of patients participating in the BIP study, 617 were found to have IFG, and 588 of them were included in the study. During the mean follow-up time of 6.2 years, DM developed in $256(44 \%)$ of the patients included, while 332 (46\%) remained without diabetes. The baseline characteristics of the patients who developed or did not develop DM are presented in Table 1. Patients who developed DM had higher BMI, higher fasting glucose levels and higher HOMA-IR than patients who did not develop DM. Patients who 
Table 1 Clinical characteristics of patients with IFG at baseline who subsequently developed or did not develop DM.

\begin{tabular}{|c|c|c|c|}
\hline Parameter & Developed DM $(n=256)$ & Did not develop DM $(n=332)$ & $\boldsymbol{P}$ \\
\hline Age (years) & $60.4 \pm 6.1$ & $61.3 \pm 6.5$ & 0.096 \\
\hline Male (\%) & 92.2 & 92.8 & 0.789 \\
\hline BMI $\left(\mathrm{kg} / \mathrm{m}^{2}\right)$ & $28.3 \pm 3.6$ & $26.7 \pm 3.2$ & $<0.001$ \\
\hline Glucose $(\mathrm{mg} / \mathrm{dl})$ & $115.8 \pm 11.1$ & $109.1 \pm 7.2$ & $<0.001$ \\
\hline HOMA-IR* & $1.5(0.9-2.4)$ & $1.3(0.8-2.0)$ & 0.012 \\
\hline $\mathrm{DBP}(\mathrm{mmHg})$ & $83.2 \pm 9.3$ & $81.2 \pm 8.6$ & 0.006 \\
\hline SBP $(\mathrm{mmHg})$ & $136.1 \pm 18.3$ & $133.6 \pm 16.7$ & 0.086 \\
\hline Triglycerides $(\mathrm{mg} / \mathrm{dl})$ & $150.5(144.1-157.3)$ & $136.0(131-141.3)$ & $<0.001$ \\
\hline Total cholesterol (mg/dl) & $214.5 \pm 18.3$ & $211.1 \pm 17.0$ & 0.023 \\
\hline $\mathrm{HDL}-\mathrm{C}(\mathrm{mg} / \mathrm{dl})$ & $33.6 \pm 5.3$ & $34.4 \pm 5.6$ & 0.094 \\
\hline LDL-C (mg/dl) & $148.8 \pm 16.6$ & $147.9 \pm 15.9$ & 0.476 \\
\hline Fibrinogen (mg/dl) & $362.1 \pm 72.9$ & $350 \pm 72.9$ & 0.045 \\
\hline WBC $\left(10^{3} / \mu \mathrm{l}\right)$ & $7.3 \pm 3.0$ & $6.9 \pm 1.55$ & 0.045 \\
\hline Insulin $(\mathrm{mU} / \mathrm{l})^{*}$ & $5.1(4.6-5.5)$ & $4.4(4.0-4.9)$ & 0.113 \\
\hline $\operatorname{CRP}(\mathrm{mg} / \mathrm{l})^{*}$ & $4.4(3.9-4.9)$ & $3.2(2.9-3.6)$ & $<0.001$ \\
\hline Adiponectin $(\mathrm{mg} / \mathrm{l})^{\star}$ & $4.1(3.9-4.4)$ & $4.6(4.3-4.9)$ & 0.009 \\
\hline
\end{tabular}

* Geometric mean (95\% confidence interval). BMI, body-mass index; HOMA-IR, homeostatic assessment of insulin resistance; DBP, diastolic blood pressure; SBP, systolic blood pressure; HDL-C, high-density lipoprotein cholesterol; LDL-C, low-density lipoprotein cholesterol; WBC, white blood cell count; CRP, C-reactive protein.

developed DM had significantly higher diastolic blood pressure than patients who did not develop DM and there was a borderline significant difference in systolic blood pressure between the groups. Triglycerides and total cholesterol levels were significantly higher in patients who developed DM than in patients who did not develop DM, but the differences in HDL-C and low-density lipoprotein cholesterol (LDL-C) levels were not statistically significant. Patients who developed DM also had significantly higher fibrinogen, white blood cell and CRP levels than patients who did not develop DM. Adiponectin levels were significantly lower at baseline in patients that developed DM: 4.1 $(3.9-4.4) \mathrm{mg} / \mathrm{l}$ vs $4.6(4.3-4.9) \mathrm{mg} / \mathrm{l}$ in the group that did not develop DM $(P=0.009)$. There were no significant differences between the groups in the following: previous history of myocardial infarction $(78.5 \%$ vs $80.4 \%)$, peripheral artery disease $(4.3 \%$ vs $3.3 \%)$ and smoking at entry (10.5\% vs $8.4 \%)$. The usage of the following medications was similar in the two groups: beta blockers $(41.8 \%$ vs $48.8 \%)$, angiotensin-converting enzyme inhibitors $(12.9 \%$ vs $15.1 \%)$, antiplatelet aggregation agents $(70.7 \%$ vs $70.5 \%)$, calcium antagonists $(50.4 \%$ vs $53.0 \%)$, nitrates $(52.7 \%$ vs $47.9 \%)$, diuretics $(16.8 \%$ vs $17.8 \%)$ and bezafibrate $(50.4 \%$ vs $52.7 \%)$. No difference in medication usage was statistically significant.

In a correlation analysis, adiponectin was positively associated with HDL-C and negatively associated with triglyceride levels (Table 2). There was also a significant but relatively weak negative correlation between adiponectin and measures of insulin sensitivity - that is, insulin and HOMA-IR. Interestingly, when we stratified patients by BMI (20), the correlation of adiponectin with insulin sensitivity was strengthened in the obese group and was no longer present in the nonobese group.

\section{Risk of subsequent DM according to baseline adiponectin quintiles}

Figure 1 shows the association of baseline adiponectin quintiles with DM development. As shown, age-adjusted increasing baseline concentration of

Table 2 Pearson correlations between baseline adiponectin levels and baseline clinical parameters.

\begin{tabular}{|c|c|c|c|c|c|c|}
\hline \multirow[b]{2}{*}{ Parameter } & \multicolumn{2}{|l|}{ All patients } & \multicolumn{2}{|l|}{$\mathrm{BMI}<30 \mathrm{~kg} / \mathrm{m}^{2}$} & \multicolumn{2}{|l|}{$\mathrm{BMI}>30 \mathrm{~kg} / \mathrm{m}^{2}$} \\
\hline & Correlation coefficient $(n=588)$ & $P$ & Correlation coefficient $(n=481)$ & $P$ & Correlation coefficient $(n=107)$ & $P$ \\
\hline Age & 0.13 & 0.002 & 0.14 & 0.002 & 0.03 & 0.75 \\
\hline BMI & -0.09 & 0.02 & -0.09 & 0.09 & -0.02 & 0.86 \\
\hline HDL-C & 0.30 & $<0.001$ & 0.29 & $<0.001$ & 0.20 & 0.002 \\
\hline Triglycerides & -0.24 & $<0.001$ & -0.25 & $<0.001$ & -0.17 & 0.07 \\
\hline Insulin & -0.09 & 0.02 & -0.04 & 0.33 & -0.29 & 0.002 \\
\hline HOMA-IR & -0.09 & 0.03 & -0.04 & 0.36 & -0.30 & 0.002 \\
\hline CRP & -0.05 & 0.30 & -0.04 & 0.35 & -0.04 & 0.63 \\
\hline
\end{tabular}

Triglycerides, insulin, HOMA-IR, CRP were log transformed. BMI, body-mass index; HDL-C, high-density lipoprotein cholesterol; HOMA-IR, homeostatic assessment of insulin resistance; CRP, C-reactive protein. 


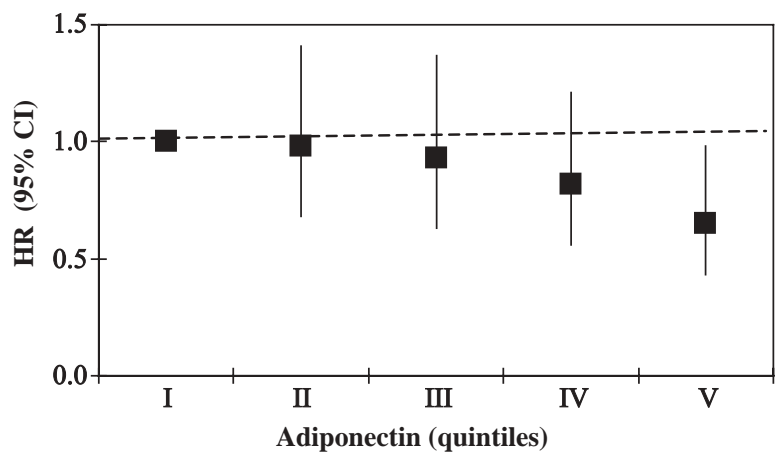

Figure 1 Age-adjusted risk of developing diabetes across quintiles of baseline adiponectin levels.

adiponectin was associated with lower risk of subsequent type 2 DM. Patients in the highest quintile of adiponectin levels, compared with patients in the lowest quintile, had a hazard ratio (HR) of 0.65 (95\% CI, 0.43-0.98). Increase of one unit (adiponectin quintile as an ordinal variable) was associated with $P=0.02$, indicating a linear trend.

With adjustment for age, an increase of 1 unit of natural logarithm of adiponectin level was associated with reduced risk of developing DM, with HR of 0.77 (95\% CI, 0.61-0.96). We tested several multivariate models for the association between diabetes development and baseline clinical variables (listed in Subjects and methods). The models that had the best performance, assessed by the goodness of fit (C-statistics), are presented in Table 3. After further adjustment for other variables, higher adiponectin levels were still associated with reduced risk to develop DM, but the statistical significance of the association was attenuated

Table 3 Multivariable analysis of the risk of developing DM.

\begin{tabular}{lccc}
\hline Variable & HR & $95 \% \mathbf{~ C l}$ & C-statistics \\
\hline Model 1 & & & 0.696 \\
$\quad$ Adiponectin & 0.83 & $0.65-1.05$ & \\
$\quad$ Fasting glucose & 1.58 & $1.45-1.71$ & \\
$\quad$ BMI & 1.20 & $1.13-1.28$ & \\
Model 2 & & & 0.697 \\
$\quad$ Adiponectin & 0.84 & $0.65-1.08$ & \\
BMl & 1.17 & $1.10-1.26$ & \\
Fasting glucose & 1.57 & $1.43-1.72$ & \\
CRP & 1.21 & $1.06-1.39$ & \\
Model 3 & & & 0.623 \\
$\quad$ Adiponectin & 0.82 & $0.64-1.04$ & \\
BMI & 1.14 & $1.06-1.21$ & \\
$\quad$ Triglycerides & 1.70 & $1.14-2.52$ & \\
CRP & 1.21 & $1.06-1.38$ & \\
\end{tabular}

Model 1 included age, adiponectin, BMI, fasting glucose. Model 2 included the same variables as in model 1 plus $\mathrm{HDL}$-cholesterol, triglycerides, HOMA-IR, CRP systolic and diastolic blood pressure. Model 3 included age, adiponectin, BMI, HDL-cholesterol, triglycerides, HOMA-IR, CRP and diastolic blood pressure. Adiponectin, triglycerides, HOMA-IR and CRP were log transformed. BMI, body-mass index; HOMA-IR, homeostatic assessment of insulin resistance; CRP, C-reactive protein.
(Table 3). As can be seen in model 1, which included BMI and fasting glucose, higher adiponectin level was associated with HR of 0.83 (95\% CI $0.65-1.05)$, and the $\mathrm{C}$-statistic of the model was 0.696. In model 2, which included also CRP, triglycerides, HDL-C and HOMA-IR, higher adiponectin level was associated with HR of 0.84 (95\% CI $0.65-1.08)$, and the C-statistic was similar to the previous model. As fasting glucose was an independent, strong predictor for developing DM in these two models, we performed model 3, which did not include fasting glucose. In this model, higher adiponectin level was associated with HR of 0.82 (95\% CI $0.64-1.04)$, and triglycerides were found to be an independent predictor of DM development, but the C-statistic of this model was reduced to 0.623 . The HR associated with adiponectin when both glucose and triglycerides were removed from the model was 0.79 (95\% CI $0.62-1.01)$ with C-statistic of 0.620 .

\section{Discussion}

Previous data from the BIP study and other studies have demonstrated that patients with coronary heart disease have a high prevalence of diabetes and abnormal fasting glucose levels (1-4). In the present study, $44 \%$ of the patients diagnosed with IFG by the newer American Diabetes Association (ADA) criteria (19) subsequently developed DM during 6.2 years of follow-up. These data agree with the high conversion rate of IFG to diabetes reported by the Diabetes Prevention Program study (5). In this study, 3234 nondiabetic persons with elevated fasting and post-load plasma glucose levels were followed for 2.8 years, and the incidence of type 2 DM was 11 per 100 person-years in the control group. The high conversion rate observed in the current study suggests that patients with known coronary heart disease and IFG represent a very highrisk group for developing type 2 DM. High fasting glucose, triglycerides, CRP and BMI, were all independent risk factors for diabetes development, and these findings confirm previous studies (4, 5, 21,22 ).

The most important finding of this study was that in patients with CAD and IFG, adiponectin levels inversely correlate with the risk of developing type $2 \mathrm{DM}$ during a 6.2-year follow-up period. Patients in the highest quintile of adiponectin levels, compared with patients in the lowest quintile, had HR of 0.65 (95\% CI, 0.43-0.98). These data suggest that even in this group of patients, who are especially prone to develop DM, adiponectin still has a protective effect of decreasing the high conversion rate to type $2 \mathrm{DM}$.

Our data as well as previous data show that low adiponectin levels are associated with features of the metabolic syndrome, a known prediabetes state (23). 
Adiponectin levels inversely correlated with triglycerides and positively with HDL-C, and in the obese group, they correlated with insulin resistance as determined by fasting insulin and HOMA-IR. Correlation studies do not distinguish cause-and-effect relationships; however several studies in rodent models support the hypothesis that adiponectin has direct insulin-sensitizing properties (10-13). Adiponectin administration was found to increase insulin sensitivity and lower circulating levels of glucose, free fatty acids and triglycerides. Adiponectin decreases hepatic glucose output and enhances muscle fat oxidation and glucose transport; these effects are mediated by activation of AMP-activated protein kinase (AMPK) (12, 13). Furthermore, in a longitudinal study in man, low adiponectin level at baseline preceded a decrease in insulin sensitivity (24).

The negative association of adiponectin levels and risk of developing type $2 \mathrm{DM}$ was shown previously in several populations (6-8). In a study of 91 Asian Indians with impaired glucose tolerance (IGT), low adiponectin level was an independent predictor of diabetes development in 1-year follow-up (25). In addition, polymorphism of the adiponectin gene predicted the conversion from IGT to type 2 diabetes in the STOP-NIDDM trial (26). These previous studies did not focus on patients with CAD. Our results not only confirm these findings but also extend them to CAD patients, a population known to have significantly lower adiponectin levels than subjects without $\operatorname{CAD}(16,27)$. Mean and median adiponectin levels in our study group were lower than reported in previous studies $(6-8,10)$, but similar to the levels reported in a recent study of patients with acute myocardial infarction, many of whom were found to have IFG and previously undiagnosed DM (3). The low range of adiponectin levels in our study may also explain the relatively moderate magnitude of the association between adiponectin and DM development. The possibility that, in this low range, the direct role of adiponectin in improving insulin sensitivity is attenuated needs to be evaluated by further studies. In addition, recent data suggest that measuring total adiponectin levels, as performed in this study and most other clinical studies, does not provide data on adiponectin complexes, and that examining the low-molecular-weight and high-molecular-weight forms may provide better correlation between adiponectin and insulin sensitivity $(12,13)$.

In conclusion, in a group of patients with CAD and IFG at baseline, 44\% developed type 2 DM during 6.2 years of follow-up. Patients who developed DM had higher BMI, fasting glucose triglyceride and CRP levels and lower adiponectin levels than patients who did not develop DM. Higher adiponectin levels were associated with a significantly reduced risk of DM development. These data suggest that even in this very highrisk group with relatively low adiponectin levels, higher adiponectin levels are still associated with reduced risk of type 2 DM development.

\section{References}

1 Ferrer M, Fulcher G, Albers CJ, Neil HA, Adams PC \& Alberti KG. Patients undergoing coronary artery bypass graft surgery are at high risk for impaired glucose tolerance and diabetes mellitus during the first postoperative year. Metabolism $1995 \mathbf{4 4}$ $1016-1027$

2 Kowalska I, Prokop J, Bachorzewska-Gajewska H, Telejko B, Kinalskal I, Kochman W \& Musial W. Disturbances of glucose metabolism in men referred for coronary arteriography. Postload glycemia as predictor for coronary atherosclerosis. Diabetes Care 200124 897-901.

3 Choi KM, Lee KW, Kim SG, Kim NH, Park CG, Seo HS, Oh DJ, Choi DS \& Baik SH. Inflammation, insulin resistance, and glucose intolerance in acute myocardial infarction patients without a previous diagnosis of diabetes mellitus. Journal of Clinical Endocrinology and Metabolism $200590175-180$.

4 Arcavi L, Behar S, Caspi A, Reshef N, Boyko V \& Knobler H. High fasting glucose levels as a predictor of worse clinical outcome in patients with coronary artery disease: results from the Bezafibrate Infarction Prevention (BIP) study. American Heart Journal 2004 $147239-245$.

5 Knowler WC, Barrett-Conner E, Fowler SE, Hamman RF, Lachin JM, Walker EA \& Nathan DM. Diabetes Prevention Program Research Group. Reduction in the incidence of type 2 diabetes with lifestyle intervention or metformin. New England Journal of Medicine 2002346 393-403.

6 Duncan BB, Schmidt MI, Pankow JS, Bang H, Couper D, Ballantyne CM, Hoogeveen RC \& Heiss G. Adiponectin and the development of type 2 diabetes. The Atherosclerosis Risk in Communities Study. Diabetes 53 2473-2478.

7 Spranger J, Kroke A, Mohlig M, Bergmann MM, Ristow M, Boeing $\mathrm{H} \&$ Pfeiffer AFH. Adiponectin and protection against type 2 diabetes mellitus. Lancet $2003361226-228$.

8 Fumeron F, Aubert R, Siddiq A, Betoulle D, Pean F, Hadjadj S, Tichet J, Wilpart E, Chesnier MC, Balkau B, Froguel P \& Marre M. Epidemiologic Data on the Insulin Resistance Syndrome (DESIR) Study Group. Diabetes $2004 \quad 53$ 1150-1157.

9 Pollin TI, Tanner K, O'Connell JR, Ott SH, Damcott CM, Shuldiner AR, McLenithan JC \& Mitchel BD. Linkage of plasma adiponectin to $3 \mathrm{q} 27$ explained by association with variation in the AMP1 gene. Diabetes $2005 \mathbf{5 4} 268-274$.

10 Diez JI \& Iglesias P. The role of the novel adipocyte-derived adiponectin in human disease. European Journal of Endocrinology 2003 $148293-300$.

11 Carling D. AMP-activated protein kinase: balancing the scales. Biochimie 200587 87-91.

12 Trujillo ME \& Scherer PE. Adiponectin - journey from an adipocyte secretory protein to biomarker of the metabolic syndrome. Journal of Internal Medicine 2005257 167-175.

13 Kadowaki T \& Yamauchi T. Adiponectin and adiponectin receptors. Endocrine Reviews 200526 439-451.

14 Kazumi T, Kawaguchi A, Hirano T \& Yoshino G. Serum adiponectin is associated with high-density lipoprotein cholesterol, triglycerides, and low-density lipoprotein particle size in young healthy men. Metabolism $200453589-593$.

15 Putz DM, Goldner WS, Bar RS, Haynes WG \& Sivitz WI. Adiponectin and C-reactive protein in obesity, type 2 diabetes, and monodrug therapy. Metabolism 200453 1454-1461.

16 Kumada M, Kihara S, Sumitsuji S, Kawamoto T, Matsumoto S, Ouchi N, Arita Y, Okamoto Y, Shinomura I, Hiraoka H, Nakamura T, Funahashi T Matsuzawa Y \& for the Osaka CAD Study Group, Association of hypoadiponectinemia with coronary 
artery disease in men. Arteriosclerosis, Thrombosis, and Vascular Biology 200323 85-89.

17 Maahs DM, Ogden LG, Kinney GL, Wadwa P, Snell-Bergeon JK Dabelea D, Hokanson JE, Ehrlich J, Eckel RH \& Rewers M. Low plasma adiponectin levels predict progression of coronary artery calcification. Circulation $2005111747-753$.

18 The Bezafibrate Infarction Prevention (BIP) Study Group, Secondary prevention by raising HDL cholesterol and reducing triglycerides in patients with coronary artery disease. Circulation 2000 $10221-27$

19 American Diabetes Association, Diagnosis and classification of diabetes mellitus. Diabetes Care 200528 (Suppl 1) S37-S42.

20 Martin LJ, Woo JG, Daniels SR, Goodman E \& Dolan LM. The relationships of adiponectin with insulin and lipids are strengthened with increasing adiposity. Journal of Clinical Endocrinology and Metabolism $2005904255-4259$.

21 Lorenzo C, Okoloise M, Williams K, Stern MP \& Haffner SM. San Antonio Heart Study. The metabolic syndrome as predictor of type 2 diabetes: the San Antonio heart Study. Diabetes Care 200326 3153-3159.

22 Kanaya AM, Fyr CL, de Rekeneire N, Shorr RI, Schwartz AV, Goodpaster BH, Newman AB, Harris T \& Barrett-Connor E. Predicting the development of diabetes in older adults: the derivation and validation of a prediction rule. Diabetes Care $2005 \mathbf{2 8}$ 404-408.

23 Mohan V, Deepa R, Pradeepa R, Vimaleswaran KS, Mohan A, Velmurugan K \& Radha V. Association of low adiponectin levels with the metabolic syndrome - the Chennai Urban Epidemiology Study (CURES-4). Metabolism 2005 54 476-481.

24 Stefan N, Vozarova B, Funahashi T, Matsuzawa Y, Weyer C, Lindsay RS, Youngren JF, Havel PJ, Pratley RE, Bogardus C \& Tataranni PA. Plasma adiponectin concentration is associated with skeletal muscle insulin receptor tyrosine phosphorylation, and low plasma concentration precedes a decrease in wholebody insulin sensitivity in humans. Diabetes $2002 \mathbf{5 1}$ $1884-1888$.

25 Snehalathatha C, Mukesh B, Simon M, Viswanathan V, Haffner SM \& Ramachandran A. Plasma adiponectin is an independent predictor of type 2 diabetes in Asian Indians. Diabetes Care $2003263226-3229$.

26 Zacharova J, Chiasson JL Laakso M \& for the STOP-NIDDM Study Group. The common polymorphism (single nucleotide $[\mathrm{SNP}]+45$ and SNP + 276) of adiponectin gene predict the conversion from impaired glucose tolerance to type 2 diabetes. The STOP-NIDDM trial. Diabetes $2005 \mathbf{5 4} 893-899$.

27 Nakamura Y, Shimada K, Fukuda D, Shimada Y, Ehara S, Hirose M, Kataoka T, Kamimori K, Shimodozono S, Kobayashi Y, Yoshiyama M, Takeuchi K \& Yoshikawa J. Implications of plasma concentrations of adiponectin in patients with coronary artery disease. Heart 200490 528-533.

Received 3 June 2005

Accepted 16 September 2005 